\title{
Effect of Extensive Interval Training on Lactate Threshold Level
}

\author{
Gopa Saha Roy ${ }^{1}$, Asish Paul, ${ }^{2, *}$, Dilip Bandopadhyyay ${ }^{3}$ \\ ${ }^{1}$ S.I.P.E.W., Hastings House, Kolkata, India \\ ${ }^{2}$ Department of Physical Education, J.U., Kolkata, India \\ ${ }^{3}$ Department of Physical Education, Kalyani University, Nadia, India \\ *Corresponding author: asp_f2000@yahoo.com
}

Received July 17, 2014; Revised July 29, 2014; Accepted August 11, 2014

\begin{abstract}
Anaerobic threshold is defined as the physiological point during exercise at which muscular lactate production exceeds the rate of lactate oxidation and as a result, lactate shows up in the system and may be balanced or not, depending on the intensity of the exercise. The purposes of the study was to determine the anaerobic thresholds level of female athletes in different distance of running. Ten female athletes, range between 22 to 24 yrs. age, having some previous experiences of training were considered as the subjects of that study. The measured variables were resting heart rate, speed for different distance and the Blood lactate. The blood lactate were measured by colorimetric method with LA Kit (Bioassay). The subjects were gone for eight-weeks scheduled extensive interval training programme. The data were collected as pre and post training manner. Significant difference has found in increased blood lactate accumulation in different gradual increase of running distance such as $250 \mathrm{mt}$., $300 \mathrm{mt}$., 350mt. and 400mt., both in pre and post training phase separately. But considering the training effect, there were the reduction (250mt.-1.98\%, 350mt.-.30\%, 400mt.-1.39\%) of blood lactate accumulation except in 300mt.( increased 2.66\%) but that were not significant in same distance of running after the extensive interval training. The conclusion may be drawn that the reasonable Extensive Interval training programme have positive impact on improvement of anaerobic threshold level reducing the blood lactate accumulation.
\end{abstract}

Keywords: anaerobic thresholds, blood lactate, interval training, extensive

Cite This Article: Gopa Saha Roy, Asish Paul, and Dilip Bandopadhyyay, "Effect of Extensive Interval Training on Lactate Threshold Level." American Journal of Sports Science and Medicine, vol. 2, no. 5A (2014): 6-9. doi: 10.12691/ajssm-2-5A-2.

\section{Introduction}

At rest and under steady-state exercise conditions, there is a balance between blood lactate production and blood lactate removal (Brooks, 2000). The lactate threshold refers to the intensity of exercise at which there is an abrupt increase in blood lactate levels (Roberts and Robergs, 1997). Although the exact Physiological factors of the lactate threshold are still being resolved, it is thought to involve the resolved; it is thought to involve the following key mechanisms (Roberts and Roberge, 1997). Although once viewed as a negative metabolic event, increased lactate production occurring exclusively during high intensity exercise as natural (Robergs, Ghiasvand, Pasker, 2004). Even at rest a small degree of lactate production takes place, which indicates there must also exists lactate removal or else there would be lactate accumulation occurring at rest. The primary means of lactate removal include its uptake by the heart, liver and kidneys metabolic fuel, (Brooks, 1985).

Ghosh A. K. (2004) expressed that anaerobic threshold is highly correlated to the distance running performance as compared to maximum aerobic capacity or $\mathrm{Vo}_{2}$ max, because sustaining a high fractional utilization of the $\mathrm{VO}_{2}$ max for a long time delays the metabolic acidosis. Tripathi and Banerjee (1992) proved that the aerobic status of the trained group was better than the untrained group in terms of ventilator anaerobic threshold in comparison with the maximal exercise performance. Gladden, 2000; Stainsby et al. 1984; 1991 defined anaerobic threshold and relates to exercise involving a large muscle mass. It is recognise that within a single muscle, glycolysis can occur, resulting in net output of lactate even at rest. Sjodin et al., 1981 onset of blood lactate accumulation, or OBLA, is defined as the intensity of exercise at which blood lactate concentration reaches $4 \mathrm{mMol}$ during an incremental exercise test. According to Karlsson, J and Jacobs (1982), Donovan, C.M. and Brooks' - G.A. (1983), Green H.J. Hughson, R.L. Orr, G.W. Ranney. D.A. (1983), Yudhin, J. and Cohen, R.D. (1975), Burke, et al (1994), Das, S. (2011) emphasized that blood lactate concentration is the net result of lactate production or appearance in the muscle and its removal from the muscle. Thus the rise in blood lactate may not necessarily indicate abrupt increase in lactate production by the exercising muscle, due to simultaneous removal process. 


\section{Materials and Methods}

The purposes of the study were to determine the anaerobic thresholds level of female athletes in different distance of running. 10 female athletes of a range between 22 to 24 yrs. age of equated groups who were volunteered as the subjects in this study. Each subject must have a minimum training age of $2-3$ years. The equated groups were comprised of close values of certain variables like age, height, body weight, resting heart rate etc. The Blood lactate was measured by colorimetric method with LA Kit (Bioassay).

\subsection{Design}

The health status of the subjects was checked at first by the institutional physician for ensuring the potential participation of the subjects in the strenuous training schedule. The group was tested on some selected variables after undergoing a two-week general conditioning programme. Then for eight-weeks, the subjects undergone scheduled extensive interval training programme. After eight-weeks training the subjects were again assessed following the same procedure by the same professionals. Statistical comparisons were done to analysis of the results obtained during Pre and Post training condition with a special reference to anaerobic threshold.

\section{Results}

The following tables show the results after statistical calculation on the collected data of different variables through the tests and measurements.

Table 1. The Mean and inter group ' $t$ '-values of blood lactate (mM/L) following different distance running before Training Programme

\begin{tabular}{|c|c|c|c|c|c|c|c|c|}
\hline & Mean & S.D. & N & Diff. & Std. Div. - Diff. & 't' value & df. & p \\
\hline $250 \mathrm{~m}$ & 8.317 & 0.468 & & & & & & \\
\hline $300 \mathrm{~m}$ & 9.636 & 0.623 & 10 & -1.319 & 0.819 & -5.094 & 9 & $0.001^{*}$ \\
\hline $350 \mathrm{~m}$ & 11.700 & 0.334 & 10 & -3.383 & 0.661 & -16.188 & 9 & $0.000^{*}$ \\
\hline $400 \mathrm{~m}$ & 10.983 & 0.444 & 10 & -2.666 & 0.809 & -10.417 & 9 & $0.000^{*}$ \\
\hline
\end{tabular}

* Significant at $\mathrm{p}<0.050$

From the above table it was found that significant difference existed in case of blood lactate concentration between the different distances of performance. The

increased up to 350mt. and then decreased. At the same time it is noted that the ' $t$ ' value is also ranging between 5.09 and 16.18 . interesting point is that the rate of mean blood lactate

Table 2. The Mean and ' $t$ '-values of blood lactate $(\mathrm{mM} / \mathrm{L})$ following different distance of running after Extensive Interval Training Programme

\begin{tabular}{|c|c|c|c|c|c|c|c|c|}
\hline & Mean & Std. Div. & N & Diff. & Std. Div. - Diff. & 't' value & df. & p \\
\hline Pre-250m & 8.317 & 0.468 & & & & & & \\
\hline $250 \mathrm{~m} '$ & 8.152 & 0.611 & 10 & 0.165 & 0.621 & 0.840 & 9 & 0.422 \\
\hline $300 \mathrm{~m} '$ & 9.892 & 0.621 & 10 & -1.575 & 0.702 & -7.094 & 9 & $0.000^{*}$ \\
\hline $350 \mathrm{~m} '$ & 11.665 & 0.642 & 10 & -3.348 & 0.855 & -12.379 & 9 & $0.000^{*}$ \\
\hline $400 \mathrm{~m} '$ & 10.830 & 0.607 & 10 & -2.513 & 0.796 & -9.986 & 9 & $0.000^{*}$ \\
\hline
\end{tabular}

* Significant at $\mathrm{p}<0.050$

From Table 2, it was observed that significant difference existed in case of blood lactate accumulation between pre-250m run and the varied distance running performance. Here it is being mentioned that mean value was also ranging between 8.15 to $11.66 \mathrm{mMl}$.
Therefore it may be concluded that there is high blood lactate accumulation in three stages at post test training condition. The highest ' $\mathrm{t}$ ' value obtained at $350 \mathrm{mtr}$ distance at the same time the minimum lactate value was recorded in $250 \mathrm{mtr}$ running performance and study supported by Banerjee, A.K. (2005).

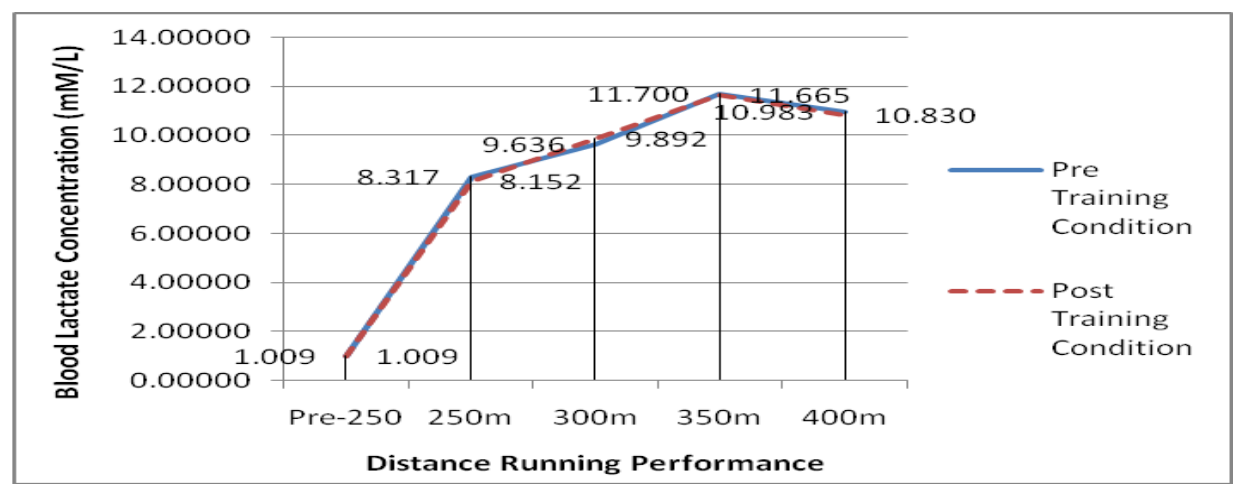

Figure 1. Graphical presentation of Mean Values of Blood Lactate of different distances in pre and post training conditions of Extensive Interval Training Group (EITG)

The probable decline of the lactate accumulation during the last phase (400 m) may further metabolic activity and to reutilize the lactate production in the blood lactate is converted as energy resource through absorption in kidney 
and liver. Therefore, it may be concluded that rapid anaerobic muscular tasks results in high accumulation of blood lactate in relation to the distances and duration of work done.

Table 3. Comparison to Pre and Post 8-weeks Extensive Interval Training effect on blood lactate on selected distance running performance

\begin{tabular}{|c|c|c|c|c|c|c|c|c|}
\hline & Mean & Std. Div. & $\mathbf{N}$ & Diff. & Std. Div. - Diff. & ' $t$ ' & df. & 'p' \\
\hline Pre-250 & 1.009 & 0.113 & & & & & & \\
\hline Pre-250' & 1.140 & 0.238 & 10 & $-0.130(12.88 \%)$ & 0.504 & 61.795 & 9 & 0.140 \\
\hline $250 \mathrm{~m}$ & 8.317 & 0.468 & & & & & & \\
\hline $250 \mathrm{~m}^{\prime}$ & 8.152 & 0.611 & 10 & 0.165 (1.98\%) & 0.621 & 0.840 & 9 & 0.422 \\
\hline $300 \mathrm{~m}$ & 9.636 & 0.623 & & & & & & \\
\hline 300m' & 9.892 & 0.621 & 10 & $-0.256(2.66 \%)$ & 1.068 & -0.758 & 9 & 0.468 \\
\hline $350 \mathrm{~m}$ & 11.700 & 0.334 & & & & & & \\
\hline 350m' & 11.665 & 0.642 & 10 & 0.035 (.30\%) & 0.766 & 0.144 & 9 & 0.888 \\
\hline $400 \mathrm{~m}$ & 10.983 & 0.444 & & & & & & \\
\hline $400 \mathrm{~m}^{\prime}$ & 10.830 & 0.607 & 10 & $0.153(1.39 \%)$ & 0.837 & 0.578 & 9 & 0.577 \\
\hline
\end{tabular}

*Significant at $\mathrm{p}<0.050$

From Table 3, it was observed that no significant difference was observed in case of blood lactate concentration between the different running of performance due to extensive interval training. Comparing between the same distances performances it was observed that the training has got some positive effect in blood lactate accumulation except the $300 \mathrm{~m}$ run but that difference were not significant.
The results of the above table reveal the rate of the blood lactate accumulation as per higher distances of running showed the diminishing trend which may predict the result of effectiveness of Extensive Interval Training, though in case of $300 \mathrm{~m}$ run not reveal as such.

During exercise of increasing distances there is a rise of blood lactate concentration and this response was first reported half a century ago (Owles, W.H. 1930, Bang, O. 1936.) and (Ghosh A. K., 2004).

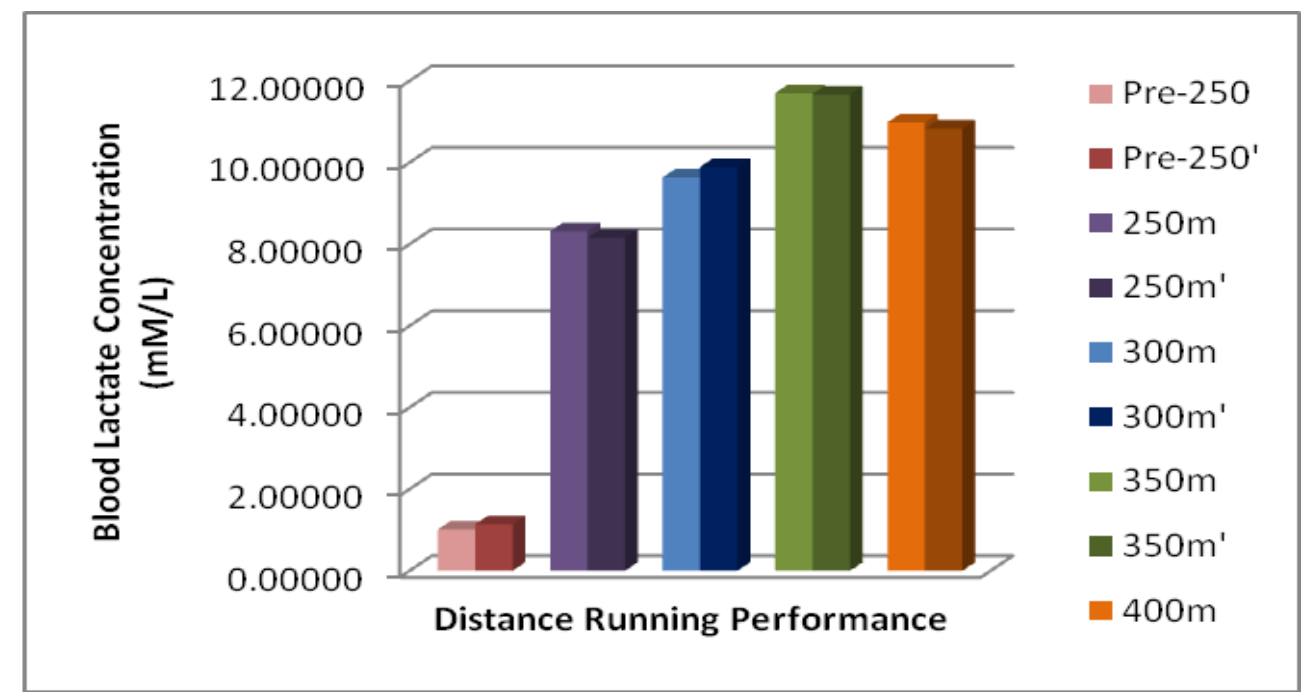

Figure 2. Graphical presentation of Mean Values of Blood Lactate of different distances in pre and post training conditions of Extensive Interval Training Group (EITG)

\section{Discussion}

Since the distance running of $250 \mathrm{~m}, 300 \mathrm{~m}, 350 \mathrm{~m}$ were conducted on the basis of the recorded time elapsed during running 400m at first, on pre and post training condition. The athletes had to run the above three phases as per recorded time for the respective distances. The athletes ran accordingly and the time was monitored by the researchers. The blood samples were collected during 250m - 400m running performances which was monitored with the intensity of work to measure the blood lactate concentration and obviously will signify the level of blood lactate threshold.
The most interesting features in the course of lactate accumulation towards $400 \mathrm{~m}$, phase by phase had given unique results. The last phase $300 \mathrm{~m}-400 \mathrm{~m}$ running rather denied further accumulation of blood lactate. This phase was subjected to a reducing trend with diminished value of blood lactate as compare to $350 \mathrm{~m}$. This is due to the utilization of a part of blood lactate for recycled energy (Brooks G. 1985, Brooks, G.H. Budouchaud, M. Brown. I. Sicurello and C. Butz, 1991; Burke, et al, 1994, Das, S., 2011).

The elevation from basal lactate value in blood is observed along with elevated intensity of muscular work which rises toward $4 \mathrm{mM} / \mathrm{L}$. The quantum of $4 \mathrm{mM}$ is considered to be the maximum value toward anaerobic threshold. The findings of the study may the value of 
$9 \mathrm{mM}$ at $250 \mathrm{~m}$ indicates the attainment of threshold level well advance of $250 \mathrm{~m}$.

The subjects of the study were heterogeneous and not under high extensive training. Hence, it is quite expected that their threshold level start earlier than $56 \mathrm{sec}$ or $250 \mathrm{~m}$.

According to the demand of this research work blood samples were collected in the pre and post training sessions and the value of blood lactate concentrations were recorded. Results showed that amount of lactate concentration reduced after the Extensive Interval Training Programme where the better results concerned to reduce the percentage of blood lactate concentration reduction took place in the Extensive Interval Training Group in the case of $400 \mathrm{~m}$ running performance. The quantum of the concentration of blood lactate at $250 \mathrm{~m}$ revealed the level of anaerobic threshold but the result showed Interval training had positive impact on anaerobic threshold elevation, which in turn shows the improvement of sports performance.

\section{Conclusion}

It may me concluded that eight weeks Extensive Interval training programme have positive impact on physiological system to raise the level of the anaerobic threshold, such an improvement of higher anaerobic threshold level has got a carryover value on sports performance.

\section{References}

[1] Bang, O., The lactate content of blood during and after muscular exercise in man. Scand. Arch. Physiol. 1936; 74: 51-82.

[2] Brooks, 1986, G.A.: The lactate shuttle during exercise, Evidence and Possible control. In: Sports Science, J Walkins, T. Reilly and L. Burwits, Eds., E and FN Spon Ltd., London 1986; 69.

[3] Burke, et al (1994), Das, S. (2011); Pattern of Blood Lactic Acid Accumulation after a short term High Intensity and a Long term low Intensity Exercise Protocol. Indian Journal of Yoga, Exercise and sports science and physical education, Vol-V, No. 1 \& 2, 2011

[4] Das, S., Pattern of Blood Lactate accumulation after a short term High Intensity and a long term low intensity exercise protocol. Indian journal of Yoga Exercise and Sport Science and Physical Education. (2011), Vol-V.

[5] Gladden, L.B. (2000). Muscle as a consumer of lactate. Med.Sci. Sports Exerc. 32:764-771.

[6] Karlsson, J., and Jacobs, (1982). Onset of blood lactate accumulation during muscular exercise as a threshold concept. Int. J. Sports Med. 3: 190-201.

[7] McPeak, C T., "Effects of an interval training programme on aerobic, anaerobic and anthropometric parameters on women". Dissertation Abstract International 33 (May. 1978): 6602-6603-A.

[8] Ready A. E and Quinney H.A. Alteration in anaerobic threshold as the result of endurance training and detraining. Med. Sci Sports Exerc 1982; 14(4), 292-296.

[9] Sjodin, B,Jacobs, I,and Karlsson, J, (1981) Onset of blood lactate accumulation and marathon running performance. Int. J. Sports Med. 2: 23-26.

[10] Stainby, W.N. Brechue,W.F., and O'Drobinak, D.M. (1991) Regulation of muscle lactate production. Med.Sci. of Sports Exerc. 23: 907-911. 